\title{
Ethanol Induces the Formation of Water-Permeable Defects in Model Bilayers of Skin Lipids
}

Romnik Thind, David W. O’Neill, Annalaura Del Regno and Rebecca Notman

Department of Chemistry and Centre for Scientific Computing, University of Warwick, Gibbet Hill Road, Coventry, CV4 7AL

*To whom correspondence should be addressed. Tel: 44 (0) 2476150889, Email: r.notman@warwick.ac.uk

\section{Electronic Supplementary Information}

Simulation Methodology .2

References.................................................................... 8

Supplementary Tables............................................................ 9

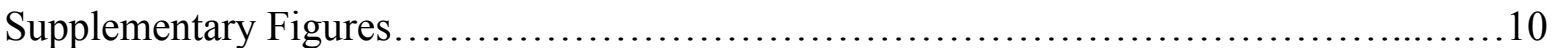




\section{Simulation Methodology}

\section{Choice of model systems}

In this work we report molecular dynamics (MD) simulations to determine the effects of ethanol $(\mathrm{EtOH})$ on the properties of ceramide 2 (CER2, ESI Figure 1) bilayers. As CER2 is one of the most abundant lipids in the skin, CER2 bilayers are a useful starting point for modelling the stratum corneum (SC) lipid layers before moving on to more complex lipid mixtures.

The SC lipids form multiple layers that fill the spaces between the corneocytes (SC skin cells) ${ }^{1}$. When a substance or formulation (e.g. cosmetic, personal care or pharmaceutical product) is applied to the skin, some of the molecules will penetrate the skin, with different formulation ingredients traversing the lipid layers at different rates before reaching the deeper layers of the skin and eventually being removed by the circulatory system ${ }^{2}$. There will also be some evaporation from the surface of the skin. The situation is further complicated by the possible influence of the ingredients on the barrier function of the skin and their interactions with each other ${ }^{2}$. Thus, bilayers at different depths will be exposed to different concentrations of the permeating molecules and determining the effective concentrations of each of the formulation ingredients in the SC is extremely challenging. Although recent progress has been made using Raman scattering microscopy ${ }^{3}$, only semi-quantitative information may be elucidated at the current time. Experiments reported in the literature have investigated the permeation of selected compounds in EtOH-containing mixtures from very low concentrations of EtOH up to pure $\mathrm{EtOH}$, for examples see Ref ${ }^{4}$. However in these studies the amount of EtOH that reaches each lipid layer is not known. Therefore in an attempt to capture the full range of possible interactions of EtOH with CER2 bilayers we 
have simulated CER2 bilayers in EtOH-water mixtures, ranging from pure water to pure EtOH in increments of $10 \mathrm{~mol} \%$.

\section{Model building}

A bilayer of 288 ceramide 2 (CER2) lipids was built by replicating the coordinates of a single CER2 molecule in a hairpin arrangement (see ESI Figure 1) to create a $12 \times 12$ monolayer and then replicating and rotating the monolayer to create a bilayer with a partially interdigitated conformation where the long chains of the CER2 molecules in one leaflet were packed end-to-end with the short chains from the other leaflet. The CER2 molecules were replicated in the $x y$ plane and the bilayer normal lay parallel to the $z$-axis of the simulation cell. The bilayer was fully hydrated by the addition of 8696 water molecules $(\approx 35$ water molecules per lipid). To equilibrate the bilayer the system was subjected to $2 \mathrm{~ns}$ of simulation (see below for parameters) with position restraints of $1000 \mathrm{~kJ} \mathrm{~mol}^{-1}$ in the $z$-direction applied to the CER2 tail atoms, followed by $2 \mathrm{~ns}$ with position restraints of $100 \mathrm{~kJ} \mathrm{~mol}^{-1}$ in the $z$ direction and then $10 \mathrm{~ns}$ of simulation without restraints. This procedure prevents disordering of the CER2 tails while the lateral spacing of the lipids reaches its equilibration value.

To generate initial configurations for the simulations with ethanol $(\mathrm{EtOH})$, the equilibrated bilayer was re-solvated with EtOH-water mixtures that had been pre-equilibrated (2 ns of $N V T$ simulation at $305 \mathrm{~K}$ followed by $2 \mathrm{~ns}$ of $N P T$ simulation at $305 \mathrm{~K}$ and 1 bar) at the required concentration. A total of 10 additional systems were prepared, where the ratio of EtOH:water ranged from $0: 10$ to $10: 0$ and the lipid:solvent ratio was 1:30. The exact molecular composition of the simulated systems is given in Table 1.

\section{Force field parameters}


The united atom force field employed for the CER2 lipids was the same as in our previous work ${ }^{5}$. To summarise, the interaction parameters for the hydrocarbon tails were taken from the force field of Berger ${ }^{6}$, which is parameterised for phospholipids and uses GROMOS87 parameters for headgroups, and specially adapted parameters for lipid tails. The atomic partial charges for the CER2 headgroups were adapted from the side chain of serine in the manner of Mombelli and co-workers ${ }^{7}$. The united atom carbons in the lipid tails had zero partial charge. The water model was SPC ${ }^{8}$. For consistency with the lipid parameters GROMOS87 parameters were used for EtOH molecules.

\section{Simulation parameters}

The bilayer simulations were carried out in the $N P T$ ensemble using the Gromacs 4.5.5 package ${ }^{9}$ and employed the Nosé-Hoover thermostat ${ }^{10}$ and a semi-isotropic pressure coupling using the Parrinello-Rahman barostat ${ }^{11}$. The simulations were carried out in the gel phase at $305 \mathrm{~K}$, which corresponds to skin temperature. All bonds in the system were constrained using the LINCS algorithm ${ }^{12}$. The time step was 2 fs. Lennard-Jones interactions were cut-off at $1.2 \mathrm{~nm}$ and the Coulombic interactions were treated using the Particle Mesh Ewald approach ${ }^{13}$, with a short-range cut-off of $1.4 \mathrm{~nm}$. Each system was simulated for between $100 \mathrm{~ns}$ and $150 \mathrm{~ns}$.

\section{Equilibration of the bilayers in EtOH-water mixtures}

As reported in the main text, EtOH molecules penetrate the CER2 bilayer and accumulate in the centre of the bilayer. To monitor the evolution of this process we plotted the EtOH density profile in the centre of the bilayer in $10 \mathrm{~ns}$ increments for each bilayer simulation. The density profiles are presented in ESI Figure 2. It can be seen that in almost all of the simulations the EtOH density profile converges by $\sim 100 \mathrm{~ns}$, after which the EtOH density 
profiles sit on top of each other or fluctuate about some average value. The exceptions are for the EtOH densities in the CER2 bilayers in 6:4, 8:2 and 9:1 EtOH:water mixtures, which still appear to be slightly increasing after $130 \mathrm{~ns}$. This could be due to processes such as lipid extraction (which facilitates the formation of larger EtOH-filled pores and thus further EtOH entry into the bilayer) occurring over longer timescales than are readily accessible in our simulations. This is supported by our observations of larger EtOH-filled pores in the bilayers in 5:5 and 7:3 EtOH:water mixtures, where the EtOH profile seems to have converged. Nevertheless we propose that our simulation times are sufficient to enable us to group our bilayer systems into three main categories; that is (i) bilayer in pure water, (ii) bilayers in EtOH:water mixtures where the ratio is low, i.e. $\leq 4: 6$ and (iii) bilayers in EtOH:water mixtures where the ratio is high, i.e., $\geq 5: 5$. By doing this we are able to draw our conclusions from multiple simulations rather than from consideration of a single simulation in isolation.

\section{Free energy calculations}

For the bilayer systems in a 0:10 and 5:5 EtOH:water mixture, the potential of mean constraint force method was used to calculate the free energy profile of water as a function of its distance to the centre of the bilayer. To generate starting configurations for the constrained MD, a steered MD simulation was carried out to pull a single water molecule from the aqueous phase into the centre of the bilayer, along the direction normal to the bilayer $(z-$ direction). A selection of configurations with the water molecule positioned at $0.1 \mathrm{~nm}$ intervals along the $z$-direction were then extracted from this trajectory and used as input for the constrained MD. A series of $4 \mathrm{~ns}$ simulations with constraints on the centre of mass distance between the bilayer and the water molecule were then carried out, giving a total of 41 simulations for the CER2 bilayer in pure water and 51 simulations for the CER2 bilayer in a 5:5 EtOH:water mixture. For each simulation the average constraint force $\langle\lambda\rangle_{z}$ was 
determined and converted into a position-dependent PMF of free energy $G(z)$ by integrating the average constraint force over all values of $z$ according to

$$
G(z)=-\int_{0}^{1}\langle\lambda\rangle_{z} d z
$$

where the limits 0 and 1 represent a difference in two states (from the bulk solvent phase to the interior of the bilayer). The convergence of the free energy profiles of the bilayer systems in a 0:10 and 5:5 EtOH:water mixture over 4 ns are shown in ESI Figures 3 and 4 respectively.

\section{Analysis}

In this section we define the different bilayer properties measured in the simulations. Unless otherwise stated, all the results reported are time averages over the last $20 \mathrm{~ns}$ of the simulation.

\section{Area per lipid}

For intact bilayers, the cross-sectional area per lipid $A_{0}$ was simply determined by calculating the area of the $x y$ plane $A$ and dividing by the number of lipids per leaflet of the bilayer $n_{\text {lipid }} / 2$. For the bilayer systems with EtOH defects or pores the area per lipid was calculated according to $A_{0}=\left(A-A_{c}\right) /\left(n_{\text {lipid }} / 2\right)$, where $A_{c}$ is the total cross-sectional area of EtOH channels in a monolayer. For each leaflet of the bilayer, $A_{c}$ was estimated using APL@Voro 14. This software calculates the cross-sectional areas of different molecules by projecting the coordinates of key atoms onto the $x y$ plane and calculating the Voronoi diagram from them. The projected area per leaflet $\left(A_{p}(L)\right)$ that corresponds to the molecule of interest is defined as the area correspondent to the Voronoi cell (or area of the defined polygon): 


$$
A_{p}(L)=\frac{1}{2} \sum_{i=0}^{N}\left(x_{i} y_{i+1}-y_{i} x_{i+1}\right)
$$

where $N$ is the number of the vertices of the Voronoi cell and $\left(x_{i}, y_{i}\right)$, the coordinates of the vertices. The molecular total projected area per leaflet of a certain species $A_{t}(L)$, described by $N$ key atoms is then equal to:

$$
A_{t}(L)=\sum_{i=0}^{N} A_{p}(i, L)=A_{c}
$$

In this work, the key atoms selected to build the Voronoi cells were the CER2 $\mathrm{N}$ atom and the EtOH $\mathrm{O}$ atom. The EtOH considered in these calculations were determined geometrically by selecting EtOH molecules within the bilayer headgroup regions. The $\mathrm{EtOH}$ in the bulk phase and absorbed to the bilayer surfaces were not considered.

Density profiles and derived quantities

To compute mass density profiles, the bilayer was divided into 100 slices in the normal direction and the number of molecules per sliced counted and normalised with respect to the volume of the slice. The bilayer thickness was estimated from the peaks in the distribution of the $\mathrm{N}$ atoms of the CER2 molecules.

\section{Hydrogen bonds}

Hydrogen bonds were defined using the geometric criterion that a hydrogen bond exists if the distance between the donor and the acceptor atoms is smaller than $0.35 \mathrm{~nm}$ and if the acceptor-donor-hydrogen angle is smaller than 60 .

$\underline{\text { Lipid tail order parameters }}$ 
The order parameters $S_{z}$ for each methyl group $\mathrm{C}_{n}$ of the lipid hydrocarbon chains, $S_{z}$, was calculated using $S_{z}=\frac{3}{2}\left\langle\cos ^{2} \theta_{z}\right\rangle-\frac{1}{2}$, where $\theta_{z}$ is the angle between the bilayer normal and the molecular axis. The molecular axis is defined as the vector from the atom $\mathrm{C}_{(n-1)}$ to $\mathrm{C}_{(n+1)}$. The brackets indicate the ensemble average. An order parameter of 1 indicates perfect alignment of the vector with the bilayer normal, $-1 / 2$ represents perfect alignment with the vector perpendicular to the normal, while a value of 0 represents isotropic behaviour.

\section{References}

1. J. Bouwstra, G. Gooris and M. Ponec, Journal of Biological Physics, 2002, 28, 211 223; L. Norlen, International Journal of Cosmetic Science, 2003, 25, 209-226.

2. C. Herkenne, I. Alberti, A. Naik, Y. Kalia, F.-X. Mathy, V. Préat and R. Guy, Pharm. Res., 2008, 25, 87-103.

3. N. A. Belsey, N. L. Garrett, L. R. Contreras-Rojas, A. J. Pickup-Gerlaugh, G. J. Price, J. Moger and R. H. Guy, J. Control. Release, 2014, 174, 37-42.

4. N. A. Megrab, A. C. Williams and B. W. Barry, Int. J. Pharm., 1995, 116, 101-112; G. Oliveira, J. Hadgraft and M. E. Lane, Int. J. Pharm., 2012, 435, 38-49; W. Russeau, J. Mitchell, J. Tetteh, M. E. Lane and J. Hadgraft, Int. J. Pharm., 2009, 374, 17-25; B. Berner, G. C. Mazzenga, J. H. Otte, R. J. Steffens, R.-H. Juang and C. D. Ebert, J. Pharm. Sci., 1989, 78, 402-407.

5. R. Notman, W. K. den Otter, M. G. Noro, W. J. Briels and J. Anwar, Biophys. J., 2007, 93, 2056-2068.

6. O. Berger, O. Edholm and F. Jahnig, Biophys. J., 1997, 72, 2002-2013.

7. E. Mombelli, R. Morris, W. Taylor and F. Fraternali, Biophys. J., 2003, 84, $1507-$ 1517.

8. H. J. C. Berendsen, J. P. M. Postma, W. F. van Gunsteren and W. F. Hermans, in Intermolecular forces, ed. B. Pullman, D. Reidel Publishing Company, Dordrecht, 1981, pp. 331-342.

9. B. Hess, C. Kutzner, D. van der Spoel and E. Lindahl, J. Chem. Theory Comput., 2008, 4, 435-447.

10. S. A. Nose, Mol. Phys., 1984, 52, 255-268; W. G. Hoover, Phys. Rev. A, 1985, 31, 1695-1697.

11. M. Parrinello and A. Rahman, J. Appl. Phys., 1981, 52, 7182-7190; S. Nose and M. L. Klein, Mol. Phys., 1983, 50, 1055-1076.

12. B. Hess, J. Chem. Theory Comput., 2008, 4, 116-122.

13. T. A. Darden, D. York and L. Pedersen, J. Chem. Phys., 1993, 98, 10089-10092; U. Essmann, L. Perera, M. L. Berkowitz, T. Darden, H. Lee and L. G. Pedersen, J. Chem. Phys., 1995, 103, 8577-8593.

14. G. Lukat, J. Krüger and B. Sommer, J. Chem. Inf. Model., 2013, 53, 2908-2925.

15. W. L. DeLano, The PyMOL Molecular Graphics System, DeLano Scientific, San Carlos, CA, USA, 2002. 
Supplementary Tables

Table 1 Composition of the solvent in the CER2 bilayer systems.

\begin{tabular}{ccc}
\hline EtOH:water & $\begin{array}{c}\text { Number of } \\
\text { ethanol } \\
\text { molecules }\end{array}$ & $\begin{array}{c}\text { Number of } \\
\text { water } \\
\text { molecules }\end{array}$ \\
\hline $0: 10$ & 0 & 8696 \\
$1: 9$ & 864 & 7776 \\
$2: 8$ & 1728 & 6912 \\
$3: 7$ & 2592 & 6048 \\
$4: 6$ & 3456 & 5184 \\
$5: 5$ & 4320 & 4320 \\
$6: 4$ & 5184 & 3456 \\
$7: 3$ & 6048 & 2592 \\
$8: 2$ & 6912 & 1728 \\
$9: 1$ & 7776 & 864 \\
$10: 0$ & 8640 & 0 \\
\hline
\end{tabular}




\section{Supplementary Figures}

nonhydroxy fatty acid (C24:0)

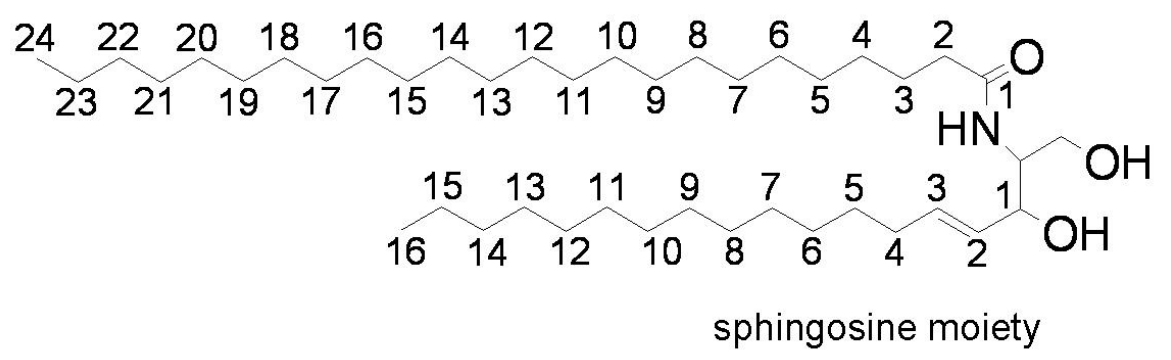

Figure 1 Molecular structure of the ceramide 2 (CER2) molecule used in this study. 

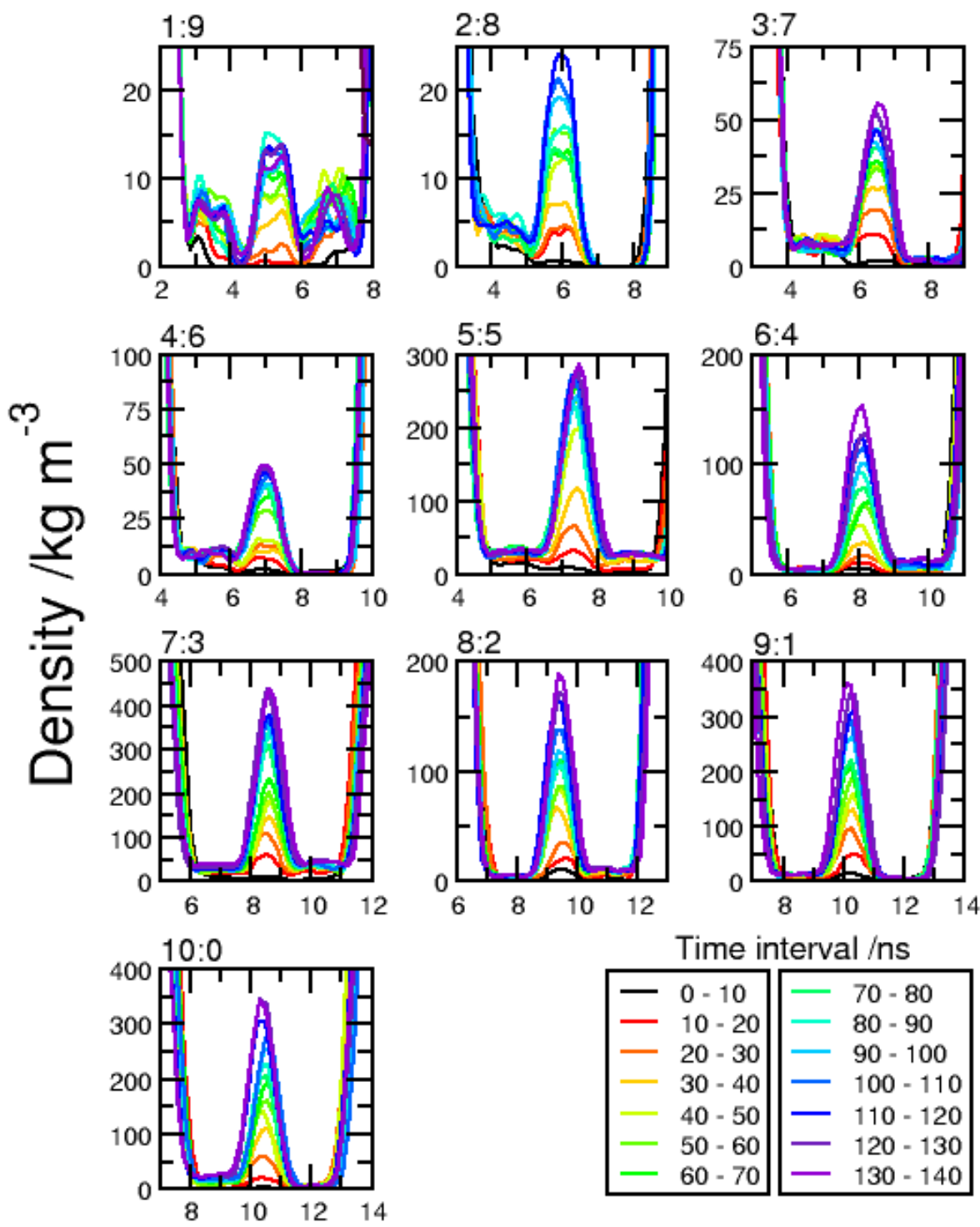

Time interval /ns

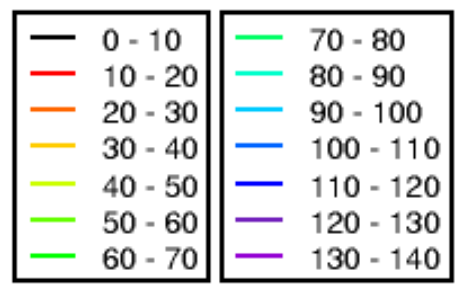

$z / \mathrm{nm}$

Figure 2 Density of EtOH in the centre of the bilayer averaged over different time intervals of the simulations. 


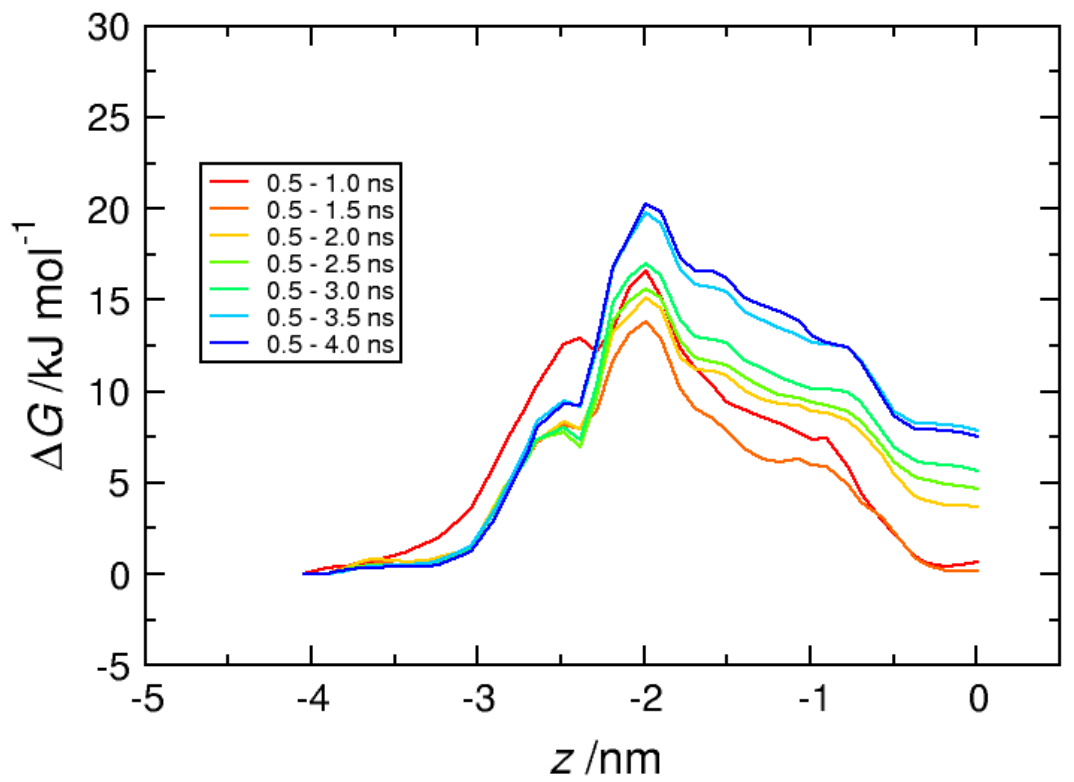

Figure 3 Convergence of the free energy profile for water in the ceramide bilayer in pure water (0:10 EtOH:water) over 4 ns of constrained MD simulations. 


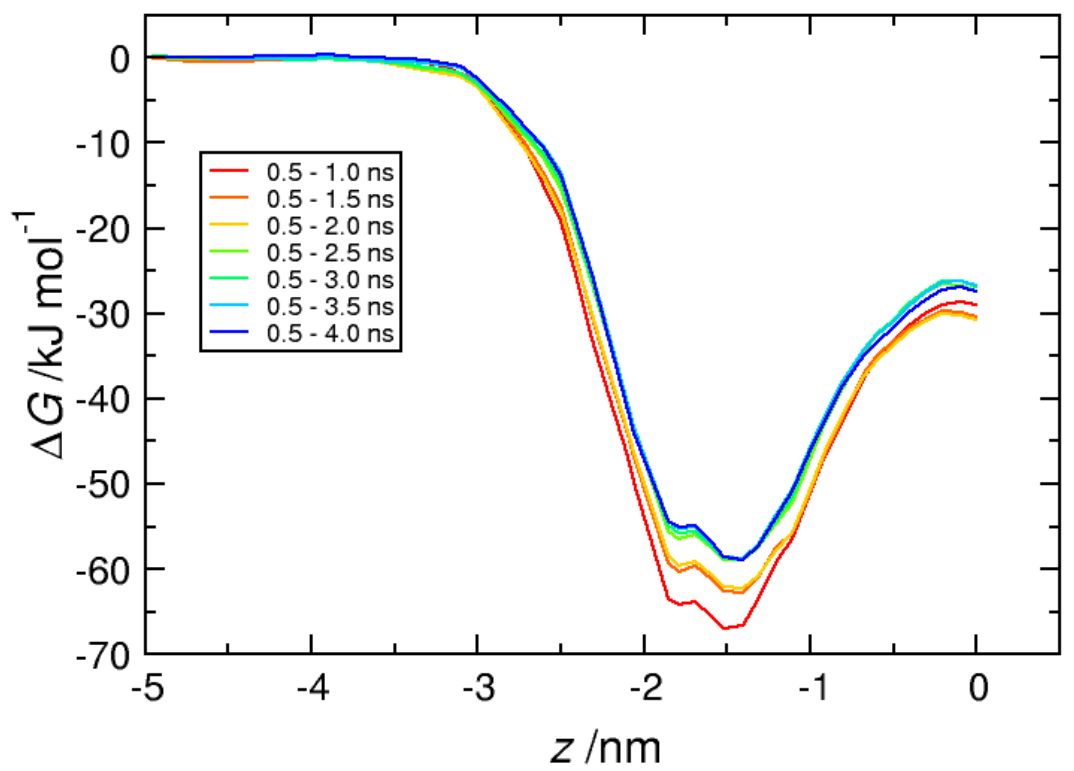

Figure 4 Convergence of the free energy profile for water in the ceramide bilayer in a 5:5 EtOH:water mixture over 4 ns of constrained MD simulations. 


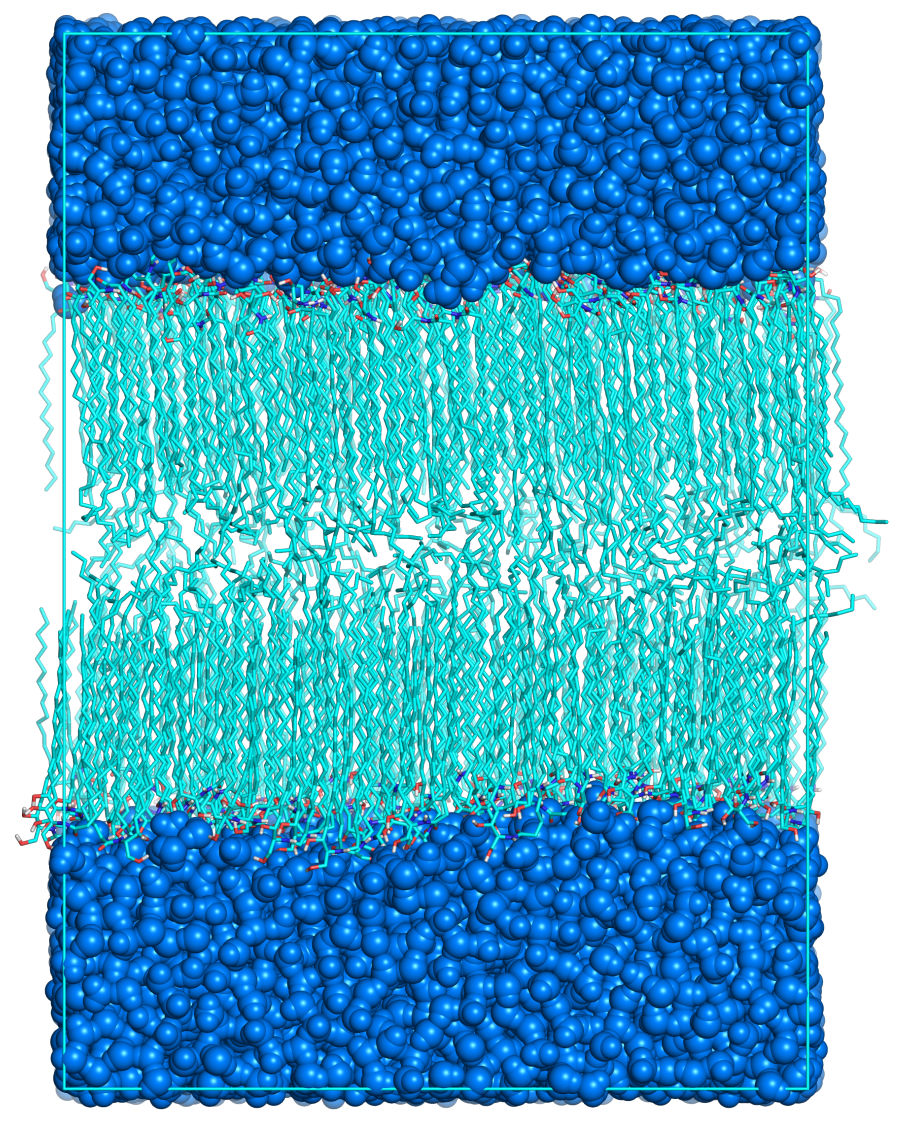

Figure 5 Snapshot of the CER2 bilayer in pure water after 100 ns of simulation. CER2 tails are shown in cyan, oxygen atoms in red, hydrogen atoms in white and nitrogen atoms in blue. Water molecules are shown in blue spacefill representation. Snapshot was generated using PyMOL ${ }^{15}$. 


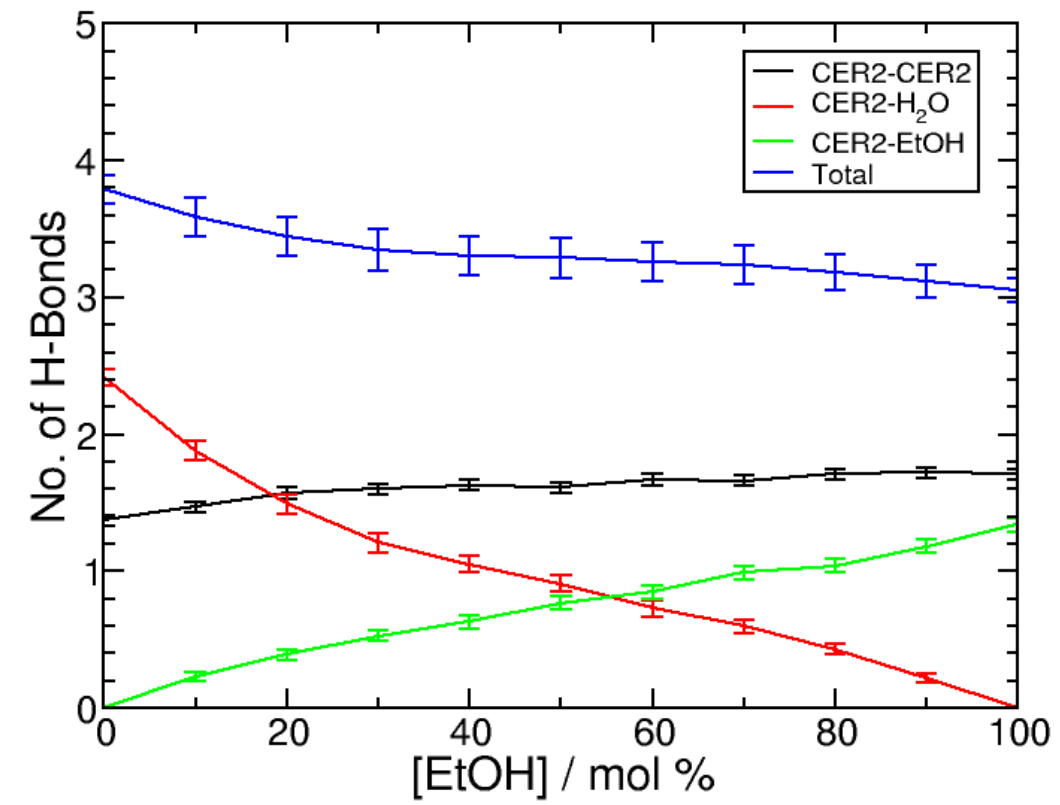

Figure 6 Number of $\mathrm{H}$-bonds between different components of the system as a function of the EtOH concentration in the solvent phase. 

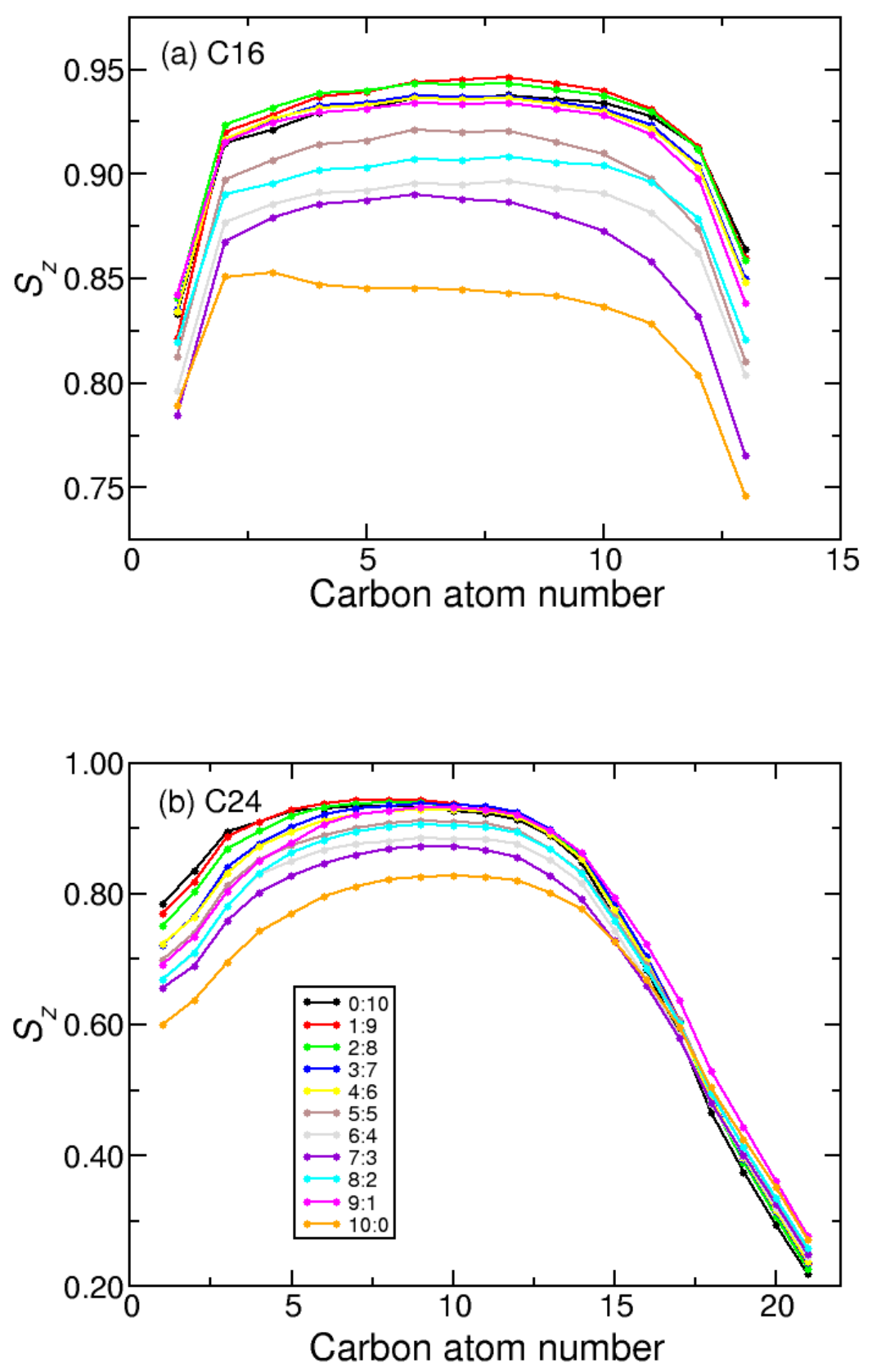

Figure 7 Lipid tail order parameters for the (a) C16 and (b) C24 chain of CER2. 


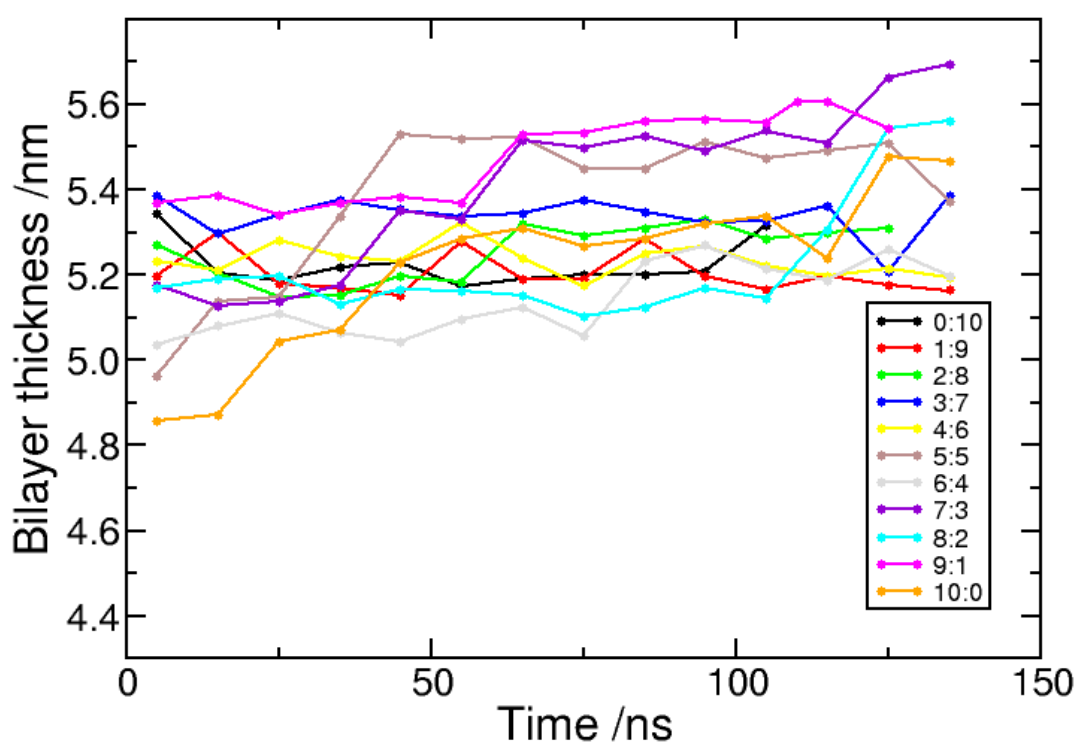

Figure 8 Thickness of the CER2 bilayer in different EtOH:water mixtures over the course of the simulation. 


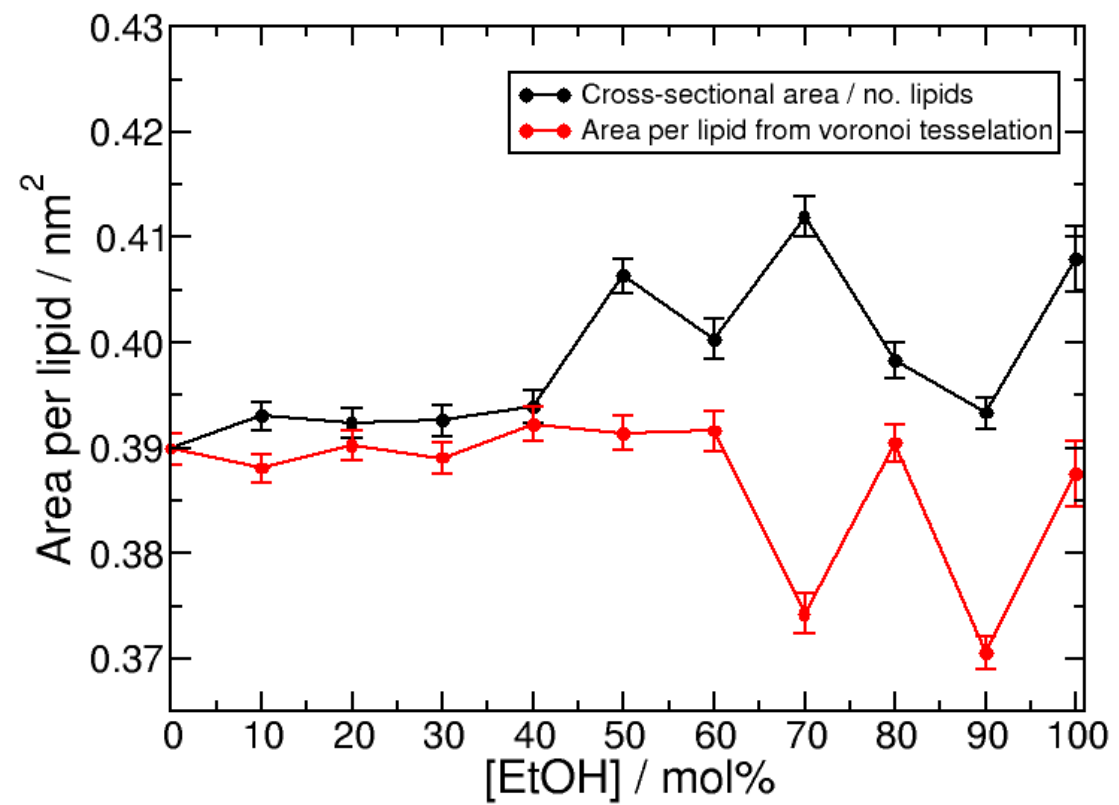

Figure 9 Area per lipid as a function of EtOH concentration estimated using (i) the cross-sectional area of the simulation cell divided by the number of CER2 molecules per leaflet and (ii) from Voronoi tessellation, which eliminates the contribution from the EtOH-filled pores. 

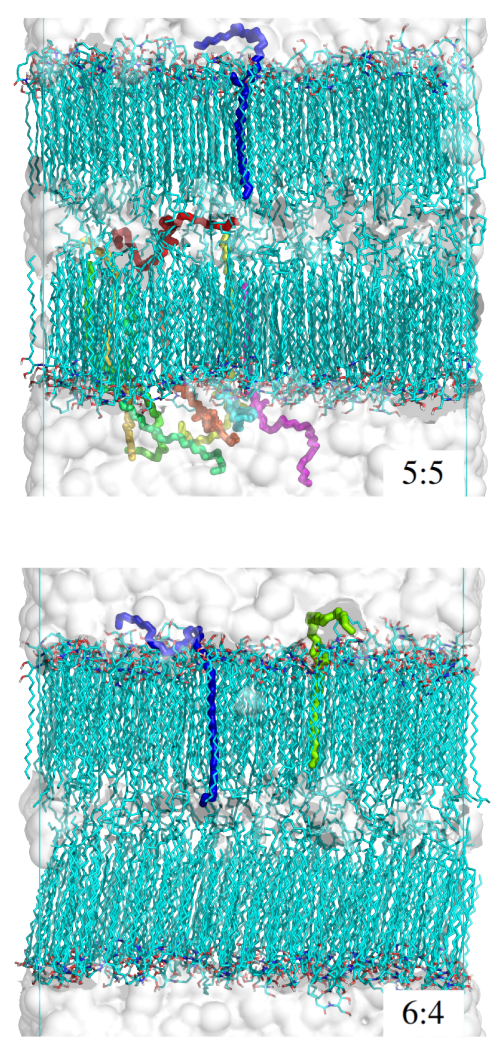
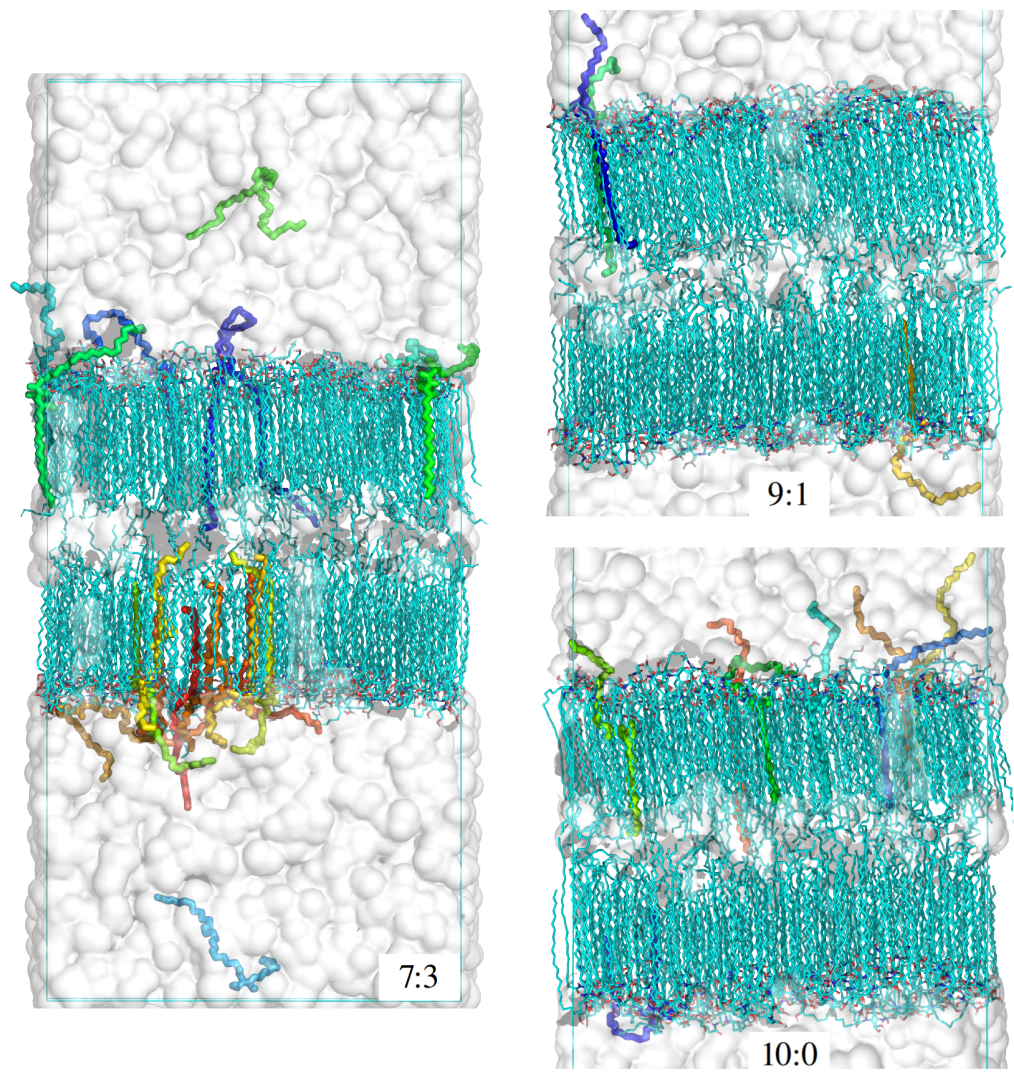

Figure 10 Snapshots of selected simulations showing partial and full lipid extraction of ceramides into the solvent. EtOH and water molecules are shown as white surfaces, CER2 molecules are shown as sticks with carbon atoms in cyan, oxygen atoms in red, nitrogen atoms in blue and hydrogen atoms in white. CER2 molecules that have been either partially (one tail) or fully (both tails) into the solvent layer are shown as thicker sticks, with each CER2 molecule coloured differently for clarity. Snapshots were generated using PyMOL ${ }^{15}$. 